\title{
Research on image preprocessing and segmentation of medical image
}

\author{
Huafeng LI \\ Shandong Medical College \\ Jinan , Shandong ,250002, China
}

\author{
Yuan ZHANG* \\ Shandong Medical College \\ Jinan, Shandong ,250002, China \\ * corresponding author
}

\begin{abstract}
-this paper presents a medical image preprocessing based on an adaptive window and the segmentation algorithm of medical image based on support vector machine, using the segmentation algorithm may be the same target which is divided into a plurality of adjacent segments. According to the pattern recognition theory, if a group of training samples can be separated by an optimal hyperplane, upper boundary of the test sample classification error rate expectations are in the training sample support vector average of the total number of training samples proportion, that reduce the influence of subjective factors, which makes segmentation result that has uniqueness.
\end{abstract}

Keywords; Medical image; Image segmentation; Adaptive window methods; Support vector machines

\section{INTRODUCTION}

Compared with other fields, such as medical image information in health field have more unique, medical image have more texture, higher resolution than ordinary images, it's correlation and storage space is greater, and to strictly ensure the reliability of clinical application, the compression, segmentation, image preprocessing, image analysis and image understanding have higher requirements.

Medical image processing across many research fields of computer, mathematics, graphics and medicine. Medical image processing include image transform, image compression, image enhancement, image smoothing, edge sharpening, image segmentation, image recognition, image fusion ${ }^{[1-3]}$. In recent years, many research and development methods is introduced and applied to medical image processing method in many areas, through continuous improvement, processing algorithm speed, treatment effect is improved in different degree.

With the rapid development and application level of computer information technology continues to improve, using computer tomography, positron emission tomography, single photon emission tomography, magnetic resonance imaging, ultrasonic imaging and the image obtained by other medical imaging equipment is widely used in each link of medical diagnosis, tissue volume, lesion location, quantitative analysis of the anatomical structure of learning, treatment planning, functional imaging data of the local body effect correction, computer guided operation and postoperative monitoring and so on ${ }^{[4]}$.

\section{RESEARCH ON MEDICAL IMAGE PREPROCESSING BASED ON ADAPTIVE WINDOW}

Medical image quality will decline to a certain extent in image acquisition, transmission and conversion process. For example, the gray value changes, noise, and geometric distortion, loss of detail that make the image information to reduce or even caused error. Therefore, the image must be on preprocessing before the image analysis and processing, which can improve the image quality. Methods of image preprocessing are commonly used image filtering, image correction, image registration and fusion etc..

A typical algorithm of adaptive window is the adaptive window based on noise distribution was proposed by W.J.PARK et al in $1999^{[5]}$, the algorithm get the relationship between image variance coefficient (standard deviation and mean ratio) and the image uniformity based on image noise model, then the algorithm will increase window or zoom Out window according to this relation judgment. In order to overcome the algorithm only uses the window edge points for statistical and did not use the window inside the pixel information and using the uniform noise distribution is used to describe the local image region distribution and other defects, Gan Rongbing ${ }^{[6]}$ put forward the distribution change detection based on adaptive window from the maximum likelihood ratio to detect different distribution, which can get expand or narrow window criterion.
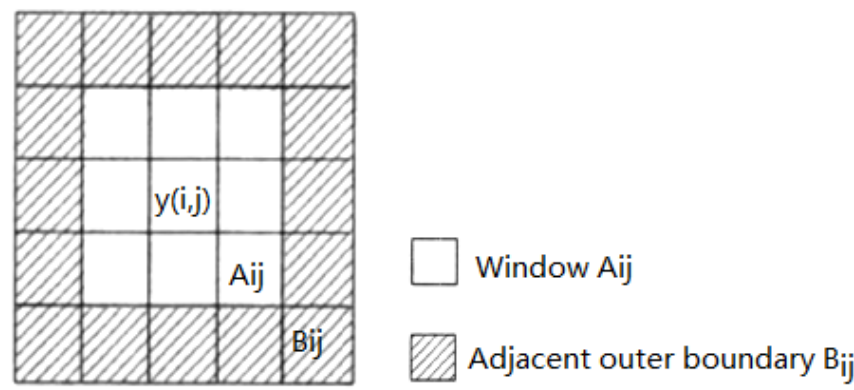

Fig.1 Schematic diagram of adaptive window

The method is determine the size of the sliding window by compared statistical characteristics of the center pixel current window and adjacent to the outer boundary. As shown in Figure 1, Aij is the current window, the Bij is outside the boundary of the current window. If the distribution of $\mathrm{Aij}$ and $\mathrm{Bij}$ are the same, $\mathrm{Bij}$ will be incorporated into the current window as the new window, otherwise it would not expand the window. Suppose that the image in a certain area is obeyed Gauss distribution ${ }^{[5]}$ : 


$$
p(y)=\frac{1}{\sqrt{2 \pi}} e^{\left[-\frac{(y-u)^{2}}{2 \sigma^{2}}\right]}
$$

$\mu$ and $\sigma$ respectively is mean and variance of $y$.

Joint probability density function of all pixels in Aij and Bij respectively is:

$$
\begin{aligned}
& p\left(Y / A_{i, j}\right)=\prod_{(i, j) e A_{i j}} p\left(y_{i, j}\right)=\prod_{(i, j) e A_{i j}} \frac{1}{\sqrt{2 \pi} \sigma_{A}} e^{\left[-\frac{\left(y_{i, j}-u_{A}\right)^{2}}{2 \sigma_{A}^{2}}\right]} \\
& =\frac{1}{\left(2 \pi \sigma_{A}^{2}\right)^{N_{A} / 2}} e^{\left[-\frac{N_{A}}{2}\right]} \\
& p\left(Y / B_{i, j}\right)=\prod_{(i, j) e B_{i j}} p\left(y_{i, j}\right)=\prod_{(i, j) e A_{i j}} \frac{1}{\sqrt{2 \pi} \sigma_{B}} e^{\left[-\frac{\left(y_{i, j}-u_{A}\right)^{2}}{2 \sigma_{B}^{2}}\right]} \\
& =\frac{1}{\left(2 \pi \sigma_{B}^{2}\right)^{N_{B} / 2}} e^{\left[-\frac{N_{B}}{2}\right]}
\end{aligned}
$$

Joint probability density function of all pixels after Aij and $\mathrm{Bij}$ be merged:

$$
\begin{aligned}
& p\left(Y / A_{i, j} \cup B_{i, j}\right)= \\
& \frac{1}{\left(2 \pi \sigma_{A B}^{2}\right)^{N_{A B} / 2}} e^{\left[-\frac{1}{2 \sigma_{A B}^{2}}\left(\sum_{(i, j) \in A_{i, j} \cup B_{i, j}}\left(y_{i, j}-\mu_{A B}\right)^{2}\right)\right]} \\
& =\frac{1}{\left(2 \pi \sigma_{A B}^{2}\right)^{N_{A B} / 2}} e^{\left[-\frac{N_{A B}}{2}\right]}
\end{aligned}
$$

$\mu_{A}, \mu_{B}, \mu_{A B}, \sigma_{A}, \sigma_{B}, \sigma_{A B}$ respectively is the mean and variance of regions Aij and $\mathrm{Bij}$, $A_{i, j} \cup B_{i, j}, N_{A}, \mathrm{~N}_{N}, N_{A B}$ are the number of pixels in each region, there is a likelihood ratio:

$$
\begin{aligned}
& R(Y)=p(Y / A) p(Y / B) / p(Y / A \cup B) \\
& =\frac{\left(\sigma_{A B}^{2}\right)^{N_{A B} / 2}}{\left(\sigma_{A}^{2}\right)^{N_{A} / 2}\left(\sigma_{B}^{2}\right)^{N_{B} / 2}}
\end{aligned}
$$

If the region of $\mathrm{Aij}$ and $\mathrm{Bij}$ are very similar, the likelihood ratio is approximate to 1 . On the other hand, the larger the likelihood ratio indicates that the distribution of two regional is different, should not be extended window. To determine the size of window and then use the filter method to estimate the center pixel. The detail flow chart is as shown in figure 2:

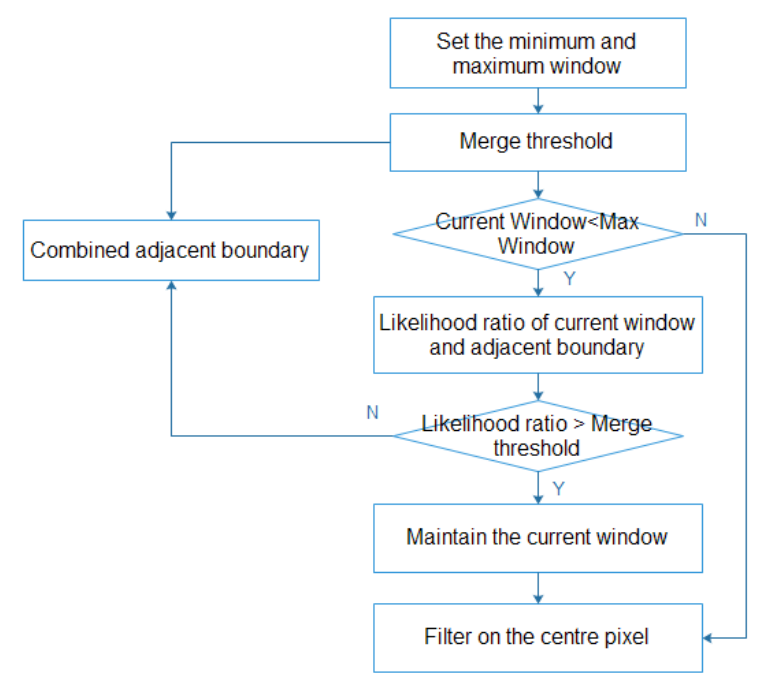

Fig.2 The flow chart of adaptive edge preserving
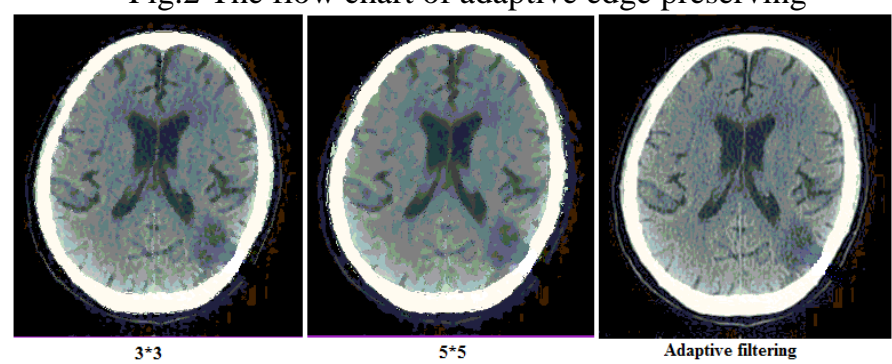

Fig.3 The result of adaptive window mean filter processing

Comparison filtering result of each filter method using adaptive window and without windows, we can see that add adaptive window can guarantee the filtering effect, and can ensure the edge and detail information. In actual use, according to the actual situation, the specific requirements of filtering effect is better still reserve the detail information selection filter rich, then add the adaptive window can better meet the requirements after treatment for the medical image filtering processing.

\section{REGION GROWING ALGORITHM FOR MEDICAL IMAGE SEGMENTATION BASED ON SUPPORT VECTOR MACHINE}

Classifier that support vector machine is similar to a neural network, which is shown in Figure 4, the output is a linear combination of several middle layer nodes, and the corresponding to each intermediate layer of nodes in the input samples and a support vector machine product, therefore is also called support vector network. 


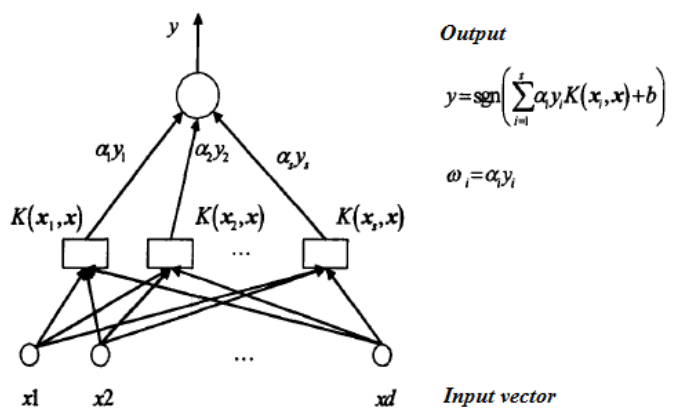

Fig.4 The diagram that support vector machine

Design segmentation algorithm in the paper is as follows:

(1) The input image be in filtering pretreatment;

(2) Using four tree segment image processing;

(3) Removing the little segmentation pixels block, and select the pixel that mean closest point in the rest of each segment, constructing a NxM area near the point which pixels is as training samples to train SVM;

(4) For each seed region, determine the seed region whether the pixel belongs to the seed by the trained SVM, one is a merger, or set aside for later judgment;

(5) Returns to step (3). (4) Continue to classify the remaining pixels, pixel until there are no classification.

Using the segmentation algorithm, there may be the same target that divided into a plurality of adjacent segment, so it must be segmentation block fusion. According to the pattern recognition theory, if a group of training samples can be separated by an optimal hyperplane, upper boundary of the test sample classification error rate expectations are in the training sample support vector average of the total number of training samples proportion, i.e.

$\mathrm{E}(\mathrm{P}($ error $)) \leq \mathrm{E}$ (Support vector number)/(Total number of training samples-1)

That is the less support vector, two types of similarity is smaller, the probability of wrong is smaller. Otherwise, the more support vectors, the classification error rate of the greater expectations, the greater the misclassification probability, the more mixed categories. When the support vector reaches a certain amount, two almost inseparable. In this paper, according to the characteristics of search, using support vector to determine whether need to two adjacent segment fusion vector machine.

\section{TEST RESULTS AND ANALYSIS}

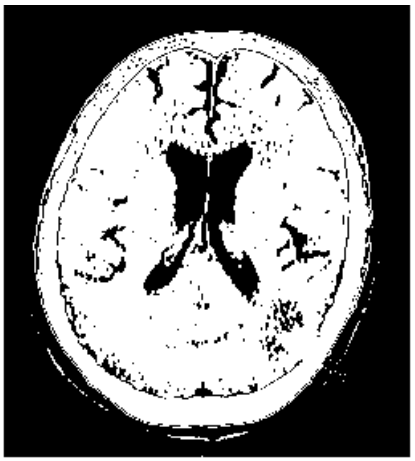

(a)

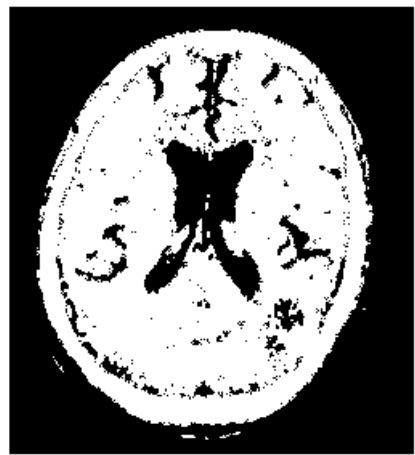

(b)
Fig.5 Comparison chart of segmentation results

As shown in Figure 5, it can be seen that the algorithm can correctly segmented out each part of information department. From the analysis of the algorithm can be seen, the algorithm does not require manual selection of seed points and growth rule, has the certain image adaptive. But as can be seen from the graph, there are some local still need further treatment.

\section{CONCLUSIONS}

For the preprocessing of DICOM images, this paper proposes the adaptive window method used in medical image filtering, using human brain images as an example, to achieve the effect of adaptive edge preserving filtering. Image segmentation is one of the key technologies for all image processing, analysis and understanding of the system. This paper gives a detailed analysis of image segmentation technology, and more comprehensive analysis of the classical image segmentation algorithm. This paper presents a segmentation algorithm of support vector machine region growing based medical image, reduce the influence of subjective factors on the segmentation results.

\section{REFERENCES}

[1] Bian X.Q, Zhang Xuegong. Pattern recognition [M]. Beijing: Tsinghua University press, 2000

[2] Hu Zhengping, Wu Yan, Zhang Hua. Research on multi-objective parallel region growing for image segmentation based on support vector learning [J]. Journal of system simulation, 2005, Vo1. 17 No. 11

[3] Hu Zhengping, Zhang Hua. Research on image segmentation algorithm that support vector learning parallel region growing combined with active contour model [J], Optical technique, 2006, Vo1.32No.3.

[4] Wang Guangjun, Tian Jin Wen, Liu Jian. Image segmentation technology based on the four fork tree structure [J]. Infrared and laser engineering, 2001, 30 (1): 12-14.

[5] Yu Jinsong, Wu Jianhua. Four tree structure in the application of complex multi-object in image segmentation [J] . Journal of Nanchang University, 2003, 25 (4): 80-82.

[6] Xue Xuejun analysis and thinking Mathematics teaching status of vocational colleges [J]. Education and Vocational,2007,21:111 -112.

[7] Lixin Ke. Several Mathematics Teaching in Vocational Institutions [J]. Zhejiang Youth College,2006,01:21 -23. 